\title{
Virus de la inmunodeficiencia humana en la era TARGA. Experiencia en un hospital de referencia
}

\author{
Human immunodeficiency virus in the intensive care \\ unit in the targa era. Experience in a referral hospital
}

Juan Higuera Lucas', Sergio Gallego Zarzosa', David Cabestrero Alonso

\begin{abstract}
Introduction: The cause of ICU admission of HIV patients has changed during the HAART era. Objective: To analyze HIV + patients admitted in the ICU of a tertiary university hospital, reference in HIV patients, during the HAART era. To describe the epidemiology of the disease in the ICU, cause of admission and comorbidities. Material and Methods: Descriptive, retrospective study. HIV + patients admitted to the ICU from May 2013 to November 2017. Collected data: HIV diagnosis, HIV related previous illnesses, previous admissions, HBV coinfection, HCV, lymphocytes, CD4, adherence to treatment, cause of admission, microbiological isolation, severity scores (SOFA, SAPS II, APACHE II), mechanical ventilation, vasoactive drugs, albumin, intra ICU mortality). Results: 1,511 patients were analyzed, 27 had previous diagnosis of $\mathrm{HIV}+, 5$ (18.9\%) were not adherent to treatment. Cause of admission: 13 patients altered level of consciousness. 7 patients acute respiratory failure. Seven patients shock. Discussion: Patients who were not adherent to treatment or coinfected with HCV-HBV had lower average of CD4, lymphocyte, albumin and hemoglobin values which were associated with higher mortality and need for vasoactive drugs. $(p<0.005)$. Non treatment adherent HIV patients admitted to the ICU with infection have higher mortality percentages than those who comply with treatment $(p<0.005)$.
\end{abstract}

\section{Key words:}

Human

immunodeficiency virus

(HIV),

intensive care (ICU),

highly active

antiretroviral therapy

(HAART)

Servicio de Medicina Intensiva del Hospital Ramón y Cajal. Madrid, España.

Fecha de recepción: 12 de junio de 2019

Fecha de aceptación: 20 de julio de 2019

\section{ORCID}

https://orcid.org/0000-0003-1778-2291

Correspondencia:

Juan Higuera Lucas.

Teléfono: 0034639618931

Dirección: Calle De Teruel №24 6E 28020 Madrid, España.

Email: jhiguera.151@gmail.com 


\section{RESUMEN}

Introducción: La causa en ingreso en UCI de los pacientes VIH ha cambiado durante la era TARGA. Objetivo: Analizar los pacientes $\mathrm{VIH}+$ que ingresan en $\mathrm{UCl}$ de un hospital terciario universitario, referencia de pacientes $\mathrm{VIH}$, durante la era TARGA. Describir la epidemiología de la enfermedad en $\mathrm{UCl}$, motivos de ingreso, comorbilidades. Material y Métodos: Estudio descriptivo, retrospectivo. Pacientes $\mathrm{VIH}+$ que ingresan en $\mathrm{UCI}$ desde mayo de 2013 hasta noviembre de 2017. Se recoge: diagnóstico $\mathrm{VIH}$, enfermedades previas relacionada, ingresos previos, coinfección VHB, VHC, linfocitos, CD4, cumplimiento de tratamiento, causa de ingreso, aislamiento microbiológico, scores de gravedad (SOFA, SAPS II, APACHE II) ventilación mecánica, fármacos vasoactivos, albúmina, mortalidad intra UCI). Resultados: Se analizan 1.511 pacientes, 27 presentan diagnóstico previo $\mathrm{VIH}+, 5(18,9 \%)$ no eran cumplidores de tratamiento. Causa de ingreso: 13 pacientes con alteración del nivel de conciencia. Siete pacientes con insuficiencia respiratoria aguda. Siete pacientes shock. Discusión: Los pacientes no cumplidores o coinfectados con VHC-VHB, presentan valores medios de CD4, linfocitos, albúmina y hemoglobina menores que se asocian a mayor mortalidad y necesidad de fármacos vasoactivos $(p<0,005)$. Los pacientes VIH no cumplidores que ingresan en $\mathrm{UCI}$ con infección, presentan porcentajes de mortalidad mayores que los cumplidores de tratamiento $(p<$ $0,005)$

\section{Palabras clave:}

Virus de la

inmunodeficiencia

humana $(\mathrm{VIH})$,

cuidados intensivos

(UCl),

tratamiento

antirretroviral de gran

actividad (TARGA)

\section{Introducción}

\section{0} millones de personas han sido infectadas por el virus de la inmunodeficiencia humana (VIH) desde el inicio de la epidemia. Treinta y cinco millones han muerto por esta causa. 36,7 millones $(30,8-42,9)$ vivían con el diagnóstico de $\mathrm{VIH}$ en el año 2016 produciéndose 1 millón de muertes relacionadas con el VIH durante ese año. El 0,8\% de los adultos de entre 15-49 años se encuentran infectados[1].

En España, se diagnosticaron 3.428 nuevos diagnósticos de VIH durante el año 2015. 39.350 nuevos diagnósticos desde el año 2003. El 83,2\% de los nuevos diagnósticos fueron entre edades comprendidas entre los 20 y 50 años[2].

El tratamiento antirretroviral de gran actividad (TARGA), ha cambiado el pronóstico y el curso a largo plazo de esta enfermedad. Ha disminuido la incidencia de enfermedades asociadas al $\mathrm{VIH}[3]$. Sin embargo, pese a que la causa de ingreso hospitalario en estos pacientes ha cambiado entre la etapa pre-TARGA y la actual, persiste siendo elevado. Este hecho se refleja también en las Unidades de Cuidados Intensivo [4], llegado a aumentar en algunas series[5],[6].

La causa principal de ingreso en las Unidades de
Cuidados Intensivos de los pacientes diagnosticados de VIH en la época pre-TARGA era insuficiencia respiratoria y sepsis con elevada incidencia de infecciones oportunistas. En la etapa post-TARGA, estos motivos de ingreso han modificado su incidencia[7]. Los factores asociados a mortalidad no han sufrido cambios significativos: Scores de gravedad (SOFA, SAPS II, APACHE II), fallo orgánico, conexión a ventilación mecánica, fallo renal, shock, albúmina o recuento de CD4[8],[9]. Estos factores, se han mantenido constantes, sin embargo, el uso de la terapia ha sido defendido en algunos estudios como factor protector[10].

La mejora en el pronóstico de estos pacientes con el desarrollo de la terapia TARGA, conlleva que la causa ingreso de estos pacientes en las Unidades de Cuidados Intensivos modifique su incidencia histórica. Nuestro objetivo es realizar un análisis de todos los pacientes $\mathrm{VIH}+$ que ingresan en la Unidad de Cuidados Intensivos de un Hospital terciario universitario, referencia en el diagnóstico y seguimiento de pacientes $\mathrm{VIH}$, durante el período en que los pacientes tienen acceso a la terapia TARGA. Describir la epidemiología de la enfermedad en la UCI, los actuales motivos de ingreso, comorbilidades y factores relacionados con la mortalidad. 


\section{Material y Métodos}

Se realiza un estudio observacional, descriptivo, retrospectivo con todos los pacientes diagnosticados de VIH que ingresan en nuestra Unidad de Cuidados Intensivos desde mayo de 2013 hasta noviembre de 2017.

Se recoge el diagnóstico de $\mathrm{VIH}$, enfermedades previas relacionadas con $\mathrm{VIH}$, ingresos en $\mathrm{UCI}$ previos, coinfección VHB, VHC, si realizaban seguimiento de su enfermedad, cumplimiento de terapia, causa de ingreso en $\mathrm{UCl}$, aislamiento microbiológico, scores de gravedad (SOFA, SAPS II, APACHE II), conexión a ventilación mecánica, necesidad de fármacos vasoactivos, albúmina, mortalidad intra UCI y hospitalaria.

Se define al paciente $\mathrm{VIH}$ como aquel al que se le diagnostica la enfermedad de forma previa a su ingreso como durante su ingreso. Se definen enfermedades previas relacionadas con $\mathrm{VIH}$ aquellas asociadas a inmunodepresión diagnosticadas en el seguimiento de su enfermedad. La coinfección VHC y VHB son aquellos pacientes que presentan pruebas de laboratorio compatibles con su diagnóstico. La causa de diagnóstico hospitalario se define como el fallo principal que justifica su ingreso en Medicina Intensiva (alteración del nivel de conciencia, fallo hemodinámico, insuficiencia respiratoria...). El aislamiento microbiológico es aquel microorganismo aislado que justifica su proceso infeccioso. Los scores de gravedad serán definidos como el máximo puntaje en las primeras 24 horas. Se recogerá el mínimo valor de albúmina durante el ingreso.

Se realiza el análisis estadístico mediante la herramienta IBM@SPSS Statistics 23. La normalidad de las variables ha sido establecida mediante el test de komogorov-Smirnov. Las variables cuantitativas con distribución normal han sido expresadas como media \pm desviación estándar (rango). Estas, han sido comparadas mediante test t-Student o ANOVA. Las distribuciones cuantitativas que no seguían una distribución normal han sido comparadas utilizando Test de Wilcoxon y son expresadas como mediana y rango intercuartílico. Las variables cualitativas son mostradas como números y porcentajes. Son comparadas mediante test de McNemar y Chi-cuadrado. El nivel de significación estadística se ha establecido con valores de $p$ menores de 0,05. Los resultados son expresados como intervalos de confianza de $95 \%$.

\section{Resultados}

Se analizan un total de 1.511 pacientes, el total de ingresos en el Servicio de Medicina Intensiva desde abril de 2014 hasta diciembre de 2017. Veintisiete pacientes presentan diagnóstico previo de virus de la inmunodeficiencia humana. El $92,6 \%$ de los casos (25/27) son hombres. Edad media 47,3 \pm 10 (25-60) años. Cuatro pacientes fueron diagnosticados de $\mathrm{VIH}$ durante 2017-2008, 8 durante 2007-1998, 11 durante $1997-1988$ y 4 1986-1988. 7,1\% de los pacientes habían presentado enfermedades relacionadas con la enfermedad previa a su ingreso y el $22,2 \%$ de los casos había requerido ingreso previo en medicina intensiva. Todos los pacientes se encontraban en seguimiento, sin embargo, $5(18,9 \%)$ no eran seguidores del tratamiento. Las cifras medias de CD4 durante su ingreso o próximo fueron de $314 \pm 231$ (10-800) y linfocitos $1.216 \pm 666$ (128-2.990). El 60\% de los pacientes (16/27) presentaron coinfección VHC o VHB.

Los índices de gravedad de los pacientes en las 24 primeras horas de ingreso fueron: SOFA 8,3 3 3,8 (117) APACHE ॥ $23,6 \pm 8,5$ (10-41) SAPS $\|$ 61,3 4 17,6 (34-95). Permanecieron ingresados una media de 7,5 $\pm 8,3$ (1-29) días. El 74\% de los pacientes (20/27) requirieron conexión a ventilación mecánica y el $63 \%$ (17/27) requirieron fármacos vasoactivos. La muestra presenta una mortalidad intra UCI de 14,8\% (4/27).

Los índices de gravedad, linfocitos, CD4+, hemoglobina y días de ingreso por subtipo de enfermo: Cumplidores de tratamiento, supervivientes y coinfección VHC-VHB, se exponen en la Tabla 1.

Trece pacientes requirieron ingreso por alteración del nivel de conciencia (3 intoxicación medicamentosa, 4 status epiléptico, 1 hemorragia intraparenquimatosa, 1 ictus isquémico, 1 meningitis, 3 encefalopatías multifactorial). Siete pacientes ingresaron por insuficiencia respiratoria aguda (1 infección orofaríngea, 2 procesos oncológicos, 3 neumonías y 1 distress secundario sepsis). Siete pacientes requirieron ingreso por shock (2 hemorragias, 4 shock séptico, 1 perforación intestinal). Se aislaron 5 gérmenes responsables de los cuadros infecciosos: 3 bacilos gram negativos, 2 cocos positivos y 1 hongo (pneumocystis) Figura 1.

El $81,1 \%$ de los pacientes seguían el tratamiento del $\mathrm{VIH}$, estos, presentaron una mortalidad intra $\mathrm{UCI}$ de $9 \%$, necesidad de DVA de $60 \%$ y VM en un $77 \%$ de los casos. Ocho casos presentaron componente infeccioso desencadenante del cuadro, entre estos se objetivó un éxitus (12,5\%).

El $18,9 \%$ de los pacientes no cumplían el tratamiento propuesto. Se objetivó una mortalidad de $40 \%$, necesidad de VM en el $80 \%$ de los casos y necesidad de fármacos vasoactivos en el $77 \%$. Se objetivaron 3 pacientes con componente infeccioso desencadenante del cuadro objetivándose 2 éxitus (67\%). 


\begin{tabular}{|c|c|c|c|c|c|c|c|c|c|}
\hline Tipo & & SOFA & SAPS II & $\begin{array}{c}\text { APACHE } \\
\text { II }\end{array}$ & CD4 & Linfocitos & Albúmina & HB & $\begin{array}{l}\text { Días de } \\
\text { ingreso }\end{array}$ \\
\hline \multirow[t]{3}{*}{ Éxitus } & No & 8,22 & 61,4 & 23,6 & 331 & 1.227 & 2,5 & 10,9 & 6,22 \\
\hline & Sí & 8,75 & 61 & 23,5 & 194 & 1.155 & 1,65 & 7,95 & 14,75 \\
\hline & $p$ & 0,8 & 0,4 & 0,9 & 0,35 & 0,8 & 0,02 & 0,07 & 0,05 \\
\hline \multirow[t]{3}{*}{ Cumplidor } & No & 10 & 67 & 30,2 & 114,5 & 850 & 2 & 9,8 & 12,6 \\
\hline & Sí & 7,91 & 60 & 22 & 354 & 1.300 & 2,5 & 10,6 & 6,3 \\
\hline & $p$ & 0,28 & 0,43 & 0,05 & 0,05 & 0,17 & 0,175 & 0,6 & 0,129 \\
\hline \multirow{3}{*}{$\begin{array}{l}\text { Coinfección } \\
\text { VHC }\end{array}$} & No & 7,7 & 58,6 & 22,8 & 357 & 1.348 & 2,6 & 11,7 & 5,82 \\
\hline & Sí & 8,7 & 63,2 & 24 & 277 & 1.126 & 2,27 & 9,6 & 8,63 \\
\hline & $p$ & 0,5 & 0,5 & 0,7 & 0,4 & 0,4 & 0,25 & 0,08 & 0,398 \\
\hline \multirow[t]{3}{*}{ VM } & No & 7 & 54,3 & 23,8 & 215 & 1.082 & 2,68 & 12,2 & 2,86 \\
\hline & $\mathrm{Si}$ & 8,75 & 63,8 & 23,5 & 347 & 1.264 & 2,3 & 9,8 & 9,1 \\
\hline & $\mathrm{p}$ & 0,3 & 0,22 & 0,9 & 0,23 & 0,54 & 0,25 & 0,08 & 0,01 \\
\hline \multirow[t]{3}{*}{ DVA } & No & 7 & 62,8 & 26,3 & 360 & 1.133 & 2,7 & 12,6 & 2,4 \\
\hline & Sí & 9 & 60,5 & 22 & 291 & 1.265 & 2,2 & 9,2 & 10,47 \\
\hline & $p$ & 0,18 & 0,74 & 0,2 & 0,5 & 0,62 & 0,04 & 0,004 & 0,002 \\
\hline
\end{tabular}

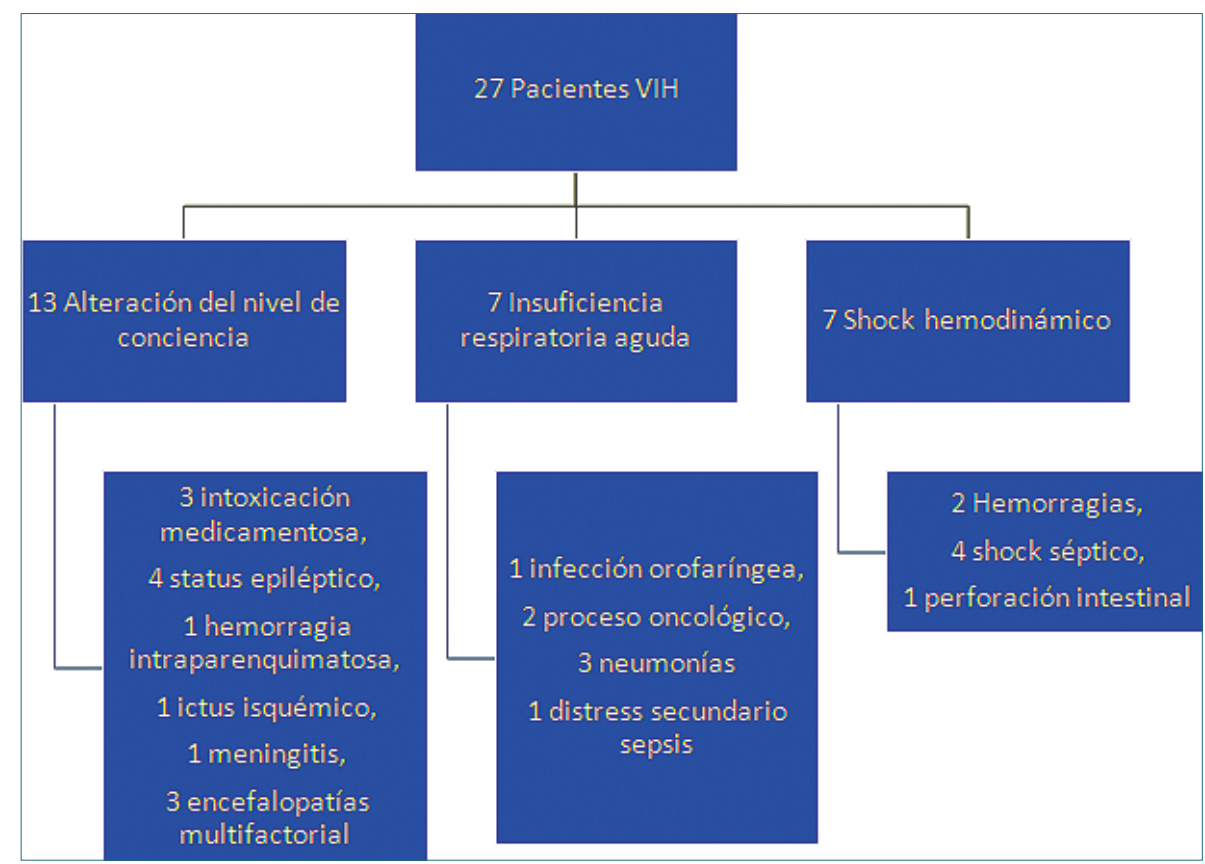

Figura 1. Se expone la división de los enfermos por motivo de ingreso en medicina intensiva. En relación al tipo de ingreso se describe el diagnóstico secundario. 
Tabla 2. Se expone el porcentaje de enfermos que requieren ventilación mecánica, fármacos vasoactivos y mortalidad por grupo de enfermo y la significación estadística

\begin{tabular}{lcccc}
\hline Tipo de paciente & & Mortalidad & Ventilación mecánica & Fármacos vasoactivos \\
Todos & & $14,8 \%$ & $74 \%$ & $63 \%$ \\
Éxitus & Sí & $100 \%$ & $100 \%$ & $100 \%$ \\
& No & $0 \%$ & $70 \%$ & $56,5 \%$ \\
& $p$ & - & 0,5 & 0,26 \\
Cumplidor tratamiento VIH & Sí & $9 \%$ & $77 \%$ & $60 \%$ \\
& No & $40 \%$ & $60 \%$ & 0,6 \\
Coinfección VHC-VHB & p & 0,14 & 0,58 & $75 \%$ \\
& Sí & $18,7 \%$ & $75 \%$ & $45 \%$ \\
Motivo de ingreso Infeccioso & No & $9 \%$ & $72,1 \%$ & 0,22 \\
& Sí & 0,62 & 0,9 & $81 \%$ \\
Motivo de ingreso infeccioso + no & Sí & $67 \%$ & $45 \%$ & $50 \%$ \\
Cumplidor & No & $6 \%$ & $93 \%$ & 0,12 \\
& No & 9,27 & 0,09 & $100 \%$ \\
\hline
\end{tabular}

En la Tabla 2 se expone la mortalidad, necesidad de ventilación mecánica y drogas vasoactivas por subtipo de enfermo.

En el análisis, se asocia también el ingreso previo en $\mathrm{UCI}$ con la hemoglobina. Los pacientes $\mathrm{VIH}$ que han ingresado en UCl previamente presentan hemoglobina media de 7,66 g/dl frente a 11,27 g/dl que presentan los pacientes que no han ingresado previamente $(p=0,008)$. También se asocia el aislamiento microbiológico con menor valor medio de albúmina: $1,7 \mathrm{~g} / \mathrm{dl}$ vs 2,61 $\mathrm{g} / \mathrm{dl}$ en aquellos pacientes sin aislamiento microbiológico ( $p=0,005)$.

\section{Discusión}

La infección por virus de la inmunodeficiencia humana fue descrita por primera vez en 1981. El perfil típico de pacientes era de hombres jóvenes con SIDA avanzado y su causa de ingreso hospitalario en Unidades de Cuidados Intensivos era principalmente infecciosa por microorganismos oportunistas como pneumocystis, toxoplasma y cryptococo. Desde el inicio de la terapia TARGA, 1996, las causas de ingreso hospitalario en pacientes $\mathrm{VIH}$, han cambiado[11]. Además, el ingreso en UCI ha aumentado debido al mejor pronóstico que presentan respecto a la era preTARGA.

Aunque se consiga una respuesta inmunovirológica adecuada, los pacientes bajo tratamiento se encuentran en riesgo de desarrollar enfermedades cardiovasculares, cáncer, enfermedad renal, enfermedades neurocognitivas e hipercoagulabilidad. Esto se debe tanto a la toxicidad derivada de los tratamientos antirretrovirales, como por la activación crónica del sistema inmune, como evidencia la elevación entre 50 y $100 \%$ de IL6 que poseen estos pacientes[12].

En nuestra muestra, la causa infecciosa, representa el $40 \%$ de los ingresos. No siendo la causa mayoritaria de ingreso, este porcentaje es notablemente más bajo que en la era pre TARGA, alcanzando en la actualidad un porcentaje similar al paciente no VIH. Las causas derivadas de la cronicidad de la enfermedad, cobran mayor peso siendo similares a los pacientes no VIH. Hemorragia intraparenquimatosa, Ictus isquémi$\mathrm{co}$, hemorragia digestiva, secundaria a proceso oncológico son algunas de ellas.

La mortalidad hospitalaria de los pacientes $\mathrm{VIH}$ ingresados en Unidades de Cuidados Intensivos se encontraba en torno al $70-90 \%$ previo a la era TARGA[13]. Dado el mal pronóstico asociado, en ocasiones, se llegó a cuestionar el ingreso de estos 
pacientes[14]. En nuestra muestra de pacientes analizados, durante la era TARGA, la mortalidad global es de $14,8 \%$. Este cambio en la mortalidad hace que la presencia de la infección no sea un impedimento de ingreso por sí mismo. Otros estudios que analizan la mortalidad de los pacientes $\mathrm{VIH}$ durante esta era, refieren también mortalidades en torno al 20-30\%[15],[16]. Estudios españoles también reportarían mortalidades similares[17], por lo que podemos afirmar que nuestros datos son más prometedores ya que la mayoría de nuestros pacientes presentaban un seguimiento preadmisión en UCI lo más optimizado posible, siendo mayores que en otros estudios españoles[17] o europeos[18],[19], alcanzando el nivel de los estadounidenses[20].

Los scores de gravedad: SOFA, APACHE II, SAPS II, son importantes predictores de mortalidad en los pacientes $\mathrm{VIH}$ [21]. En nuestra muestra, no hemos encontrado significación estadística en la diferencia de medias. El valor de albúmina y CD4, se asocia también a mayor mortalidad en los pacientes $\mathrm{VIH}[22]$. En nuestra muestra, los pacientes que sobreviven a su ingreso en $\mathrm{UCl}$, presentan valores medios de CD4 de $331 \mathrm{u} /$ $\mathrm{mm}^{3}$ mientras que los no supervivientes presentan valores medios de $194 \mathrm{u} / \mathrm{mm}^{3}$. El valor de albúmina medio es de $1,65 \mathrm{~g} / \mathrm{dl}$ en los pacientes que no sobreviven mientras que es de $2,5 \mathrm{~g} / \mathrm{dl}$ en los supervivientes $(p=0,02)$. El valor de hemoglobina, se ha asociado también a mayor mortalidad. En nuestra muestra se asocia a mayor uso de fármacos vasoactivos junto con la albúmina $(p<0,05)$. En nuestra muestra, los pacientes $\mathrm{VIH}$ con ingresos previos en $\mathrm{UCl}$, presentan valores de hemoglobina menores que aquellos que lo hacen por primera vez. 7,66 g/dl frente a $11,7 \mathrm{~g} / \mathrm{dl}(\mathrm{p}$ $=0,008$ ). Estos datos, sitúan la caquexia como un elemento fundamental a la hora de establecer el pronóstico de estos pacientes. Seguimiento del tratamiento (reflejado en número de CD4) y estado nutricional basal (reflejados en albúmina, hemoglobina...) son los parámetros que se asocian a un mejor pronóstico.

También se asocia el aislamiento microbiológico con menor valor medio de albúmina: 1,7 g/dl vs 2,61 $\mathrm{g} / \mathrm{dl}$ en aquellos pacientes sin aislamiento microbiológico $(p=0,005)$.

En el análisis por subgrupos, los pacientes no cumplidores del tratamiento TARGA previo a su ingreso presentan una media de días de ingreso mayores que los cumplidores. También se ha asociado esto a los pacientes con coinfección VHC-VHB. La media de estancia es también mayor en los pacientes VIH que requieren fármacos vasoactivos, ventilación mecánica y los registrados como éxitus $(p<0,005)$. Estos datos, sugieren que la gravedad de la patología, requiriendo mayor soporte, conlleva estancias mayores, similar a los pacientes no $\mathrm{VIH}$. Sin embargo, en igualdad de condiciones, los pacientes no cumplidores, requieren más días de estancia en UCI.

La necesidad de intubación orotraqueal, es factor independiente de mortalidad en los pacientes que requieren ingreso en medicina intensiva. Esto también se refleja en los pacientes $\mathrm{VIH}[23]$. Los pacientes registrados en nuestro estudio, precisan ventilación mecánica en un $74 \%$ de los casos (20/27). Se objetiva una mortalidad de $20 \%$ en estos pacientes. Aquellos pacientes que no precisan intubación orotraqueal y conexión a ventilación mecánica presentan una supervivencia de $100 \%$. Diecisiete pacientes requieren fármacos vasoactivos, presentando una mortalidad de $23,5 \%$. La necesidad de fármacos vasoactivos también se asocia a mayor mortalidad en pacientes $\mathrm{VIH}$.

Durante el período de tiempo en el que los pacientes tienen acceso a la terapia TARGA, el ingreso en las Unidades de Cuidados Intensivos ha aumentado. La causa de ingreso infecciosa ha disminuido su incidencia y los pacientes tienen mejor evolución en parte por presentar valores CD4 medios más altos. Chiang HH., en la revista critical care[24] analiza la mortalidad de los pacientes VIH sépticos y no sépticos, sus valores de CD4 y la mortalidad. Se objetiva que aquellos diagnosticados de sepsis con valores de CD4 menores, presentan mayor mortalidad. En nuestra muestra, hay pacientes que no cumplen el tratamiento propuesto tras el diagnóstico de VIH. Estos pacientes, presentan valores de CD4, linfocitos, albúmina y hemoglobina menores que los pacientes que sí lo cumplen. Además, presentan porcentaje de mortalidad mayores a $40 \%$ vs $9 \%$. Cuando el motivo de ingreso de los pacientes no cumplidores es infeccioso la mortalidad de estos es de $66 \%$ vs $9 \%$ en aquellos pacientes que no cumplen estas características ( $p=0,049)$.

La insuficiencia respiratoria es una causa común de ingreso de los pacientes $\mathrm{VIH}$ en $\mathrm{UCl}$. La bibliografía refiere que este motivo implica un $25-40 \%$ de las causas[25]. En nuestra muestra, representa el 26\%. De estas, $5 / 7$ el $71 \%$ son secundarias a proceso infeccioso. La neumonía por pneumocystis se diagnosticaba en alrededor del $25 \%$ de los pacientes $\mathrm{VIH}$ ingresados en medicina intensiva durante los años 90, llegando a registrarse un $50 \%$ en algunas series[26]. Ahora, en nuestro estudio, sólo se ha aislado en una ocasión en un paciente sin tratamiento previo. La introducción del tratamiento con corticoides adyuvante a finales de los años 80, ha reducido la mortalidad de esta infección[27]. Actualmente, la principal causa de ingreso por sepsis respiratoria es de causa bacteriana[28].

Los pacientes VIH ingresados en medicina intensi- 
va por shock séptico o sepsis, presentan mayor mortalidad que los pacientes no VIH. La mortalidad de los pacientes VIH ingresados por shock séptico es mayor que la de los pacientes VIH ingresados por otras causas llegando a multiplicar su incidencia por tres[29]. En nuestro medio, la mortalidad de los pacientes VIH diagnosticados de proceso infeccioso es de $27 \%$ vs $6 \%$ en aquellos no infectados. La mortalidad de los pacientes con infección y VIH no seguidores de tratamiento presentan una mortalidad de $66 \%$. La causa infecciosa se postula por tanto como un importante factor de morbilidad asociado al VIH, sin embargo, el correcto tratamiento TARGA, disminuye sensiblemente la mortalidad de los mismos, haciendo que lleguen con mayores cifras de linfocitos y CD4, y por tanto, una inmunidad endógena más eficaz. Existen al respecto evidencias del beneficio de no discontinuar el TARGA si se ha llegado a una supresión virológica eficaz al ingresar en $\mathrm{UCl}$, si la condición del paciente lo permite[30],[31].

La enfermedad hepática, es la comorbilidad más frecuente en nuestra muestra, lo que coincide con otros estudios similares. La coinfección por VHC O VHB es frecuente en los pacientes VIH llegando a ser del 35-60\% en algunas series[17],[32]. En nuestra muestra la coinfección está presente en el $60 \%$ de los casos. Estos pacientes presentan valores medios de CD4, linfocitos, albúmina y hemoglobina menores. La bibliografía refiere peor pronóstico en estos pacientes[32]. Nuestras diferencias con respecto a la mortalidad han sido de $18,7 \%$ en los pacientes con coinfección. Esto es congruente con otros estudios españoles, en los que la coinfección aumenta la mortalidad intra-UCI. Probablemente la infección VIH acelere la historia natural del VHC, ya que el estado proinflamatorio en el que estos pacientes se hallan sumidos se sume a la inflamación hepática y aumente aún más el estado de inflamación descontrolada que sucede durante la sepsis.

El estudio cuenta con la limitación de ser observacional, retrospectivo y unicéntrico. Sin embargo, cuenta con la ventaja de que se ha realizado en un hospital referencia en $\mathrm{VIH}$ analizando todos los pacientes ingresados en medicina intensiva con esta patología en era TARGA.

\section{Conclusiones}

Podemos concluir, por tanto, que los avances en el tratamiento del VIH que se han llevado a cabo en la era TARGA han permitido abrir las puertas a los pacientes infectados, aproximando su media de supervivencia a los no infectados en una $\mathrm{UCI}$ como la nuestra, y disminuyendo el peso de las infecciones oportunistas entre las causas de ingreso. Sin embargo, se observan más causas de ingreso indirectamente relacionadas con el VIH y la inflamación crónica que esto conlleva.

Asimismo, los pacientes cumplidores de tratamiento llegaban con mejor puntaje en las escalas pronósticas y presentaban mayor supervivencia global, siendo deletérea la combinación VIH/NHC.

Nuestros resultados son congruentes con los que muestran otras series de nuestro país, por lo que se hace necesario realizar estudios multicéntricos que nos permitan objetivar mejor el perfil de los pacientes $\mathrm{VIH}$ que recibimos en las UCls en la actualidad.

\section{Referencias}

1. World health organization. http://www.who.int/gho/hiv/en/

2. Ministerio de Sanidad. Servicios sociales e igualdad. https://www. msssi.gob.es/ciudadanos/enfLesiones/enfTransmisibles/sida/vigilancia/InformeVIH_SIDA_2016. pdf

3. Huang L, Quartin A, Jones D, Havlir DV. Intensive care of patients with HIV infection. N Engl J Med. 2006 Jul;355(2):173-81. https:// doi.org/10.1056/NEJMra050836
PMID:16837681

4. Chiang $\mathrm{HH}$, Hung $\mathrm{CC}$, Lee $\mathrm{CM}$, Chen HY, Chen MY, Sheng WH, et al. Admissions to intensive care unit of HIV-infected patients in the era of highly active antiretroviral therapy: etiology and prognostic factors. Crit Care. 2011 Aug;15(4):R202. https://doi.org/10.1186/cc10419 PMID:21871086

5. Nuesch R, Geigy N, Schaedler E, Battegay M. Effect of highly active antiretroviral therapy on hospitalization characteristics of HIV-infected patients. Eur J Clin Microbiol Infect Dis. 2002 Sep;21(9):684-7. https://doi. org/10.1007/s10096-002-07923 PMID:12373504

6. Vincent B, Timsit JF, Auburtin $M$, Schortgen $F$, Bouadma L, Wolff $M$, et al. Characteristics and outcomes of HIV-infected patients in the ICU: impact of the highly active antiretroviral treatment era. Intensive Care Med. 2004 May;30(5):859-66. https://doi.org/10.1007/s00134004-2158-z PMID:14767592 
7. Casalino E, Wolff M, Ravaud P, Choquet $C$, Bruneel F, Regnier B. Impact of HAART advent on admission patterns and survival in HIV-infected patients admitted to an intensive care unit. AIDS. 2004 Jul;18(10):1429-33. https://doi.org/10.1097/01. aids.0000131301.55204.a7 PMID:15199319

8. Barbier F, Coquet I, Legriel $S$, Pavie J, Darmon M, Mayaux J, et al. Etiologies and outcome of acute respiratory failure in HIVinfected patients. Intensive Care Med. 2009 Oct;35(10):1678-86. https://doi.org/10.1007/s00134009-1559-4 PMID:19575179

9. Coquet I, Pavie J, Palmer P, Barbier F, Legriel S, Mayaux J, et al. Survival trends in critically ill HIVinfected patients in the highly active antiretroviral therapy era. Crit Care. 2010;14(3):R107. https://doi.org/10.1186/cc9056 PMID:20534139

10. Croda J, Croda MG, Neves A, De Sousa dos Santos S. Benefit of antiretroviral therapy on survival of human immunodeficiency virus-infected patients admitted to an intensive care unit. Crit Care Med. 2009 May;37(5):160511. https://doi.org/10.1097/ CCM.0b013e31819da8c7 PMID:19325488

11. Powell K, Davis JL, Morris AM, Chi A, Bensley MR, Huang L. Survival for patients With HIV admitted to the ICU continues to improve in the current era of combination antiretroviral therapy. Chest. 2009 Jan;135(1):117. https://doi.org/10.1378/ chest.08-0980 PMID:18719058

13. Akgün KM, Miller RF. Critical Care in Human Immunodeficiency Virus-Infected Patients. Semin Respir Crit Care Med. 2016 Apr;37(2):303-17. https://doi. org/10.1055/s-0036-1572561 PMID:26974306

14. Sarkar P, Rasheed HF. Clini- cal review: respiratory failure in HIV-infected patients-a changing picture. Crit Care. 2013 Jun; 17(3):228. https:// doi.org/10.1186/cc12552 PMID:23806117

15. Akgün KM, Tate JP, Pisani $M$, Fried $T$, Butt $A A$, Gibert $C L$, et al. Medical ICU admission diagnoses and outcomes in human immunodeficiency virusinfected and virus-uninfected veterans in the combination antiretroviral era. Crit Care Med. 2013 Jun;41(6):145867. https://doi.org/10.1097/ CCM.0b013e31827caa46 PMID:23507717

16. Barbier F, Roux A, Canet $E$, Martel-Samb P, Aegerter P, Wolff $M$, et al. Temporal trends in critical events complicating HIV infection: 1999-2010 multicentre cohort study in France. Intensive Care Med. 2014 Dec;40(12):1906-15. https://doi. org/10.1007/s00134-014-34817 PMID:25236542

17. P. Vidal-Cortésa, L.A. ÁlvarezRochab, P. Fernández-Ugidosa, M.A. Pérez-Velosoa, I.M. SuárezPaulc, A. Virgós-Pedreirad, S. Pértega-Díaze, Á.C. CastroIglesiasf. Epidemiology and outcome of HIV-infected patients admitted to the ICU in the Current highly active antiretroviral therapy era. DOI: 10.1016/j. medin.2019.02.012

18. Turtle L, Vyakernam R, MenonJohansson A, Nelson MR, Soni $\mathrm{N}$. Intensive care usage by HIVpositive patients in the HAART era. Interdiscip Perspect Infect Dis. 2011;2011:847835. https:// doi.org/10.1155/2011/847835 PMID:22121360

19. Foo H, Clezy K, Post JJ. The long-term outcome of HIVinfected patients after intensive care admission. Int J STD AIDS. 2012 Jun;23(6):e4-8. https://doi. org/10.1258/ijsa.2009.009341
PMID:22807552

20. Akgün $K M$, Tate JP, Pisani $M$, Fried T, Butt AA, Gibert $C L$, et al. Medical ICU admission diagnoses and outcomes in human immunodeficiency virusinfected and virus-uninfected veterans in the combination antiretroviral era. Crit Care Med. 2013 Jun;41(6):145867. https://doi.org/10.1097/ CCM.0b013e31827caa46 PMID:23507717

21. Nickas G, Wachter RM. Outcomes of intensive care for patients with human immunodeficiency virus infection. Arch Intern Med. 2000 Feb;160(4):541-7. https://doi.org/10.1001/archinte.160.4.541 PMID:10695695

22. Chiang $\mathrm{HH}$, Hung $\mathrm{CC}$, Lee $\mathrm{CM}$, Chen HY, Chen MY, Sheng WH, et al. Admissions to intensive care unit of HIV-infected patients in the era of highly active antiretroviral therapy: etiology and prognostic factors. Crit Care. 2011 Aug;15(4):R202. https://doi.org/10.1186/cc10419 PMID:21871086

23. Nickas G, Wachter RM. Outcomes of intensive care for patients with human immunodeficiency virus infection. Arch Intern Med. 2000 Feb;160(4):541-7. https://doi.org/10.1001/archinte.160.4.541 PMID:10695695

24. Chiang $\mathrm{HH}$, Hung $\mathrm{CC}$, Lee $\mathrm{CM}$, Chen HY, Chen MY, Sheng WH, et al. Admissions to intensive care unit of HIV-infected patients in the era of highly active antiretroviral therapy: etiology and prognostic factors. Crit Care. 2011 Aug;15(4):R202. https://doi.org/10.1186/cc10419 PMID:21871086

25. Leifeld L, Rockstroh J, Skaide S, Wasmuth JC, Altfeld M, Paar $W D$, et al. Indication, outcome and follow up of intensive care in patients with HIV-infection. Eur J Med Res. 2000 May;5(5):199- 
202. PMID:10806121

26. Alves C, Nicolás JM, Miró JM, Torres A, Agustì C, Gonzalez J, et al. Reappraisal of the aetiology and prognostic factors of severe acute respiratory failure in HIV patients. Eur Respir J. 2001 Jan;17(1):87-93. https://doi.org/1 0.1183/09031936.01.17100870 PMID:11307762

27. Bozzette SA, Sattler FR, Chiu J, Wu AW, Gluckstein D, Kemper C, et al.; California Collaborative Treatment Group. A controlled trial of early adjunctive treatment with corticosteroids for Pneumocystis carinii pneumonia in the acquired immunodeficiency syndrome. N Engl J Med. 1990 Nov;323(21):14517. https://doi.org/10.1056/ NEJM199011223232104 PMID:2233917

28. Adlakha A1. Pavlou M, Walker
DA, Copas AJ, Dufty N, Batson S, Edwards SG, Singer M, Miller RF. Survival of HIV infected patients in the intensive care unit in the era of highly active antiretroviral therapy. Int J STD AIDS. 2011 Sep;22(9):498-504. https://doi. org/10.1258/ijsa.2011.010496 PMID:21890545

29. Japiassú AM, Amâncio RT, Mesquita EC, Medeiros DM, Bernal $H B$, Nunes EP, et al. Sepsis is a major determinant of outcome in critically ill HIV/AIDS patients. Crit Care. 2010;14(4):R152. https://doi.org/10.1186/cc9221 PMID:20698966

30. Meybeck A, Lecomte L, Valette M, Van Grunderbeeck $N$, Boussekey N, Chiche A, et al. Should highly active antiretroviral therapy be prescribed in critically ill HIVinfected patients during the
ICU stay? A retrospective cohort study. AIDS Res Ther. 2012 Sep;9(1):27. https://doi. org/10.1186/1742-6405-9-27 PMID:23020962

31. Akgün KM, Huang $L$, Morris $A$, Justice $A C$, Pisani $M$, Crothers K. Critical illness in HIV-infected patients in the era of combination antiretroviral therapy. Proc Am Thorac Soc. 2011 Jun;8(3):301-7. https:// doi.org/10.1513/pats.201009060WR PMID:21653532

32. Medrano J, Álvaro-Meca A, Boyer $A$, Jiménez-Sousa MA, Resino $S$. Mortality of patients infected with HIV in the intensive care unit (2005 through 2010): significant role of chronic hepatitis $C$ and severe sepsis. Crit Care. 2014 Aug;18(4):475. https://doi. org/10.1186/s13054-014-04753 PMID:25159592 